\title{
A Prospective Study to Evaluate the Prevalence of Infection in the Environment of ICU of a Tertiary Care Government Hospital
}

\author{
Shakti Datt Sharma', Vandana Chugh', Rakesh Kumar $^{3}$ \\ ${ }^{1}$ Anaesthesiology, Senior Administrative Grade Medical Officer, Dr Baba Saheb Ambedkar \\ Hospital and Medical College, Delhi. \\ ${ }^{2}$ Anesthesiology, Specialist Anaesthesia, Dr Baba Saheb Ambedkar Hospita and Medical College, Delhi. \\ ${ }^{3}$ Anaesthesiology, Director Professor, Maulana Azad Medical College and Associated Lok Nayak Hospital, New Delhi. \\ DOI: https://doi.org/10.24321/2349.7181.201806
}

Abstract
Introduction: The presence of microorganisms with acquired resistance to multiple antibiotics complicates
the management and outcome of critically ill patients. In the intensive care units (ICUs), this can lead to
increased mortality. A remarkable increase in microbial resistance aiming selected pathogens has been
reported over time. Exposure to antimicrobial agents has been thought to be an important contributor
to its development. The environment that can contribute to this spread of infection can be of any kind,
e.g., equipment, water, mobile phones, sink, taps, etc. Planning for containment and control of hospital-
acquired infection (HAI) has to be based on the knowledge of possible sources. Thus we planned this study
in the hospital.
Methods: A prospective study was conducted, in which surveillance of the ICU environment and personnel,
was carried out. Swabs were collected from 13 different sites from the environment (sinks, respirators,
medicine trolleys, bed sheets, etc.) and healthcare workers twice a week throughout the duration of the
study. The swabs were inoculated into a transport media and processed in the department of microbiology.
Data was collected as per reporting received from the microbiology department. Compiled data was
presented as actual number and percentage.
Result: Two hundred thirteen (63\%) of 338 samples collected no growth. All the site samples were infected
except the disinfectant solutions and the doctors. Escherichia Coli was the commonest organism grown
(74.4\%), $12 \%$ grew Klebsiella, $4.8 \%$ showed Pseudomonas, $4.8 \%$ coagulase negative staph, $4 \%$ showed Staph
aureus. All 52 water samples were positive for E. coli. The prevalence of microorganisms was maximum in
the water (100\%) and quite high in the sinks.
Conclusion: The ICU environment seems to be a potential reservoir for pathogens. Therefore, strict adherence
to environmental infection control measures is essential to prevent healthcare-associated infections.
Keywords: Environment, ICU, Infection

Corresponding Author: Dr. Vandana Chugh, Dr Baba Saheb Ambedkar Hospita and Medical College, Delhi.

E-mail Id: deepakvandana786@yahoo.co.in

Orcid Id: https://orcid.org/0000-0002-0412-2211

How to cite this article: Sharma SD, Chugh V, Kumar R. A Prospective Study to Evaluate the Prevalence of Infection in the Environment of ICU of a Tertiary Care Government Hospital. J Adv Res Med 2018; 5(2): 1-5. 


\section{Introduction}

Infection control in an intensive care unit (ICU) can be a challenging task for any physician. More than 5 million patients are admitted per year, to an ICU, in the United States. ${ }^{1,2}$

ICU-related infections increase the length of hospital stay, cost of hospitalization; mortality and morbidity is increased due to ICU related infections. ${ }^{3}$

Antimicrobial-resistant pathogens, confronted in the highest numbers in the ICUs within the hospitals are a pervasive problem and affect the clinical outcome of the patients admitted. Over time, there has been a progressive increase in antimicrobial resistance among pathogens in ICU worldwide. This fact has been corroborated by various reports like that of former National Nosocomial Infection Surveillance System. Results of studies at the National Healthcare Safety Network confirm a higher occurrence rate for most antimicrobial-resistant pathogens in ICU patients with hospital-acquired infections when compared to non-ICU patients. ${ }^{4}$

Exposure to antimicrobial agents has been thought to be an important contributor to antimicrobial resistance among pathogens. Gastrointestinal carriage, the main reservoir of these organisms in healthcare settings, is associated with a high risk of self- and cross-infections. In addition, the environment and colonized patients are continuous exogenous sources from which other patients can be colonized via the transitorily colonized hands of healthcare workers. Environmental spread through equipment, water sinks, etc., can contribute to infection in ICU. Planning for control of HAl has to be based on the knowledge of possible sources. This is the basis for our study.

\section{Aims and Objectives}

- To find out the prevalence of infections in the ICU environment

- To recommend measures to decrease HAI in ICU

\section{Materials and Methods}

\section{Sample Design}

Prospective study.

\section{Sample Size}

Sample size was such as to ensure a minimum of 30 isolates from swabs of environment in the Intensive Care Unit.

\section{Sites for Collecting Samples}

Swabs were collected from 13 different sites - taps $\times 2$, sinks $\times 2$, water $\times 2$, ventilators, medicine trays, bed rails, disinfectants, stethoscopes, doctors and nurses, twice a week throughout the duration of the study.

\section{Specimen Collection}

Moistened swabs were rubbed on the tap knobs, medicine trolleys, ventilators, bed rails or diaphragms of stethoscopes. In case of water and disinfectant sampling, the swabs were dipped in these liquids (running water or the disinfectant bottle/container without touching the tap or container. In case of doctors and nurses, moistened swabs were rubbed on the palms, web spaces and nail beds. The swabs were inoculated into a transport media and processed in the microbiology department.

\section{Sample Processing}

Environmental swabs were sent to the microbiology department and were processed as per protocol. Samples were inoculated on to Mac Conkey agar and blood agar and incubated at $37^{\circ} \mathrm{C}$ for $18 \mathrm{~h}$. The isolated bacteria were identified on colony morphology, biochemical characteristics and confirmed by slide agglutination, where required, as per standard guidelines.

\section{Data Collection}

The presence or absence of any microorganism growth was reported by the microbiology department. In case the sample was positive for some growth, the nature of microorganism was mentioned by the microbiology department. The information was noted on a proforma and then, at the end of study, compiled in a master chart.

\section{Results}

In this study, we collected a total of 338 environmental samples from 13 different sites over 13 weeks and sent them to microbiology department for processing. Out of these 338 samples, 213 (63\%) showed no growth and remaining $37 \%$ showed growth of various organisms (Table 1). All the sites sampled were infected except the disinfectant solutions and doctors. The prevalence of microorganism was maximum in water (100\%) and quite high in the sinks (86.5\%). E. coli was the commonest organism grown and of the infected samples, $74 \%$ of the infected samples grew E. Coli, $12 \%$ grew Klebsiella, $4.8 \%$ showed Pseudomonas, $4.8 \%$ coagulase negative staph, $4 \%$ showed $S$. aureus. All 52 water samples were positive for $E$. Coli which was also found on the tap knobs and sinks. Klebsiella was second commonest organism and was seen in $12 \%$ of the infected samples. It was seen in both the positive samples picked from the nurses and $29 \%$ of the samples from the two sinks. The other relatively low occurrence organisms were pseudomonas and coagulase negative S. Aureus (4.8\% each of positive samples). While Pseudomonas was grown from samples from sinks, medical trays and ventilators, coagulase-negative staphylococcus was found on samples from ventilators, bed rails and diaphragm of stethoscopes. 


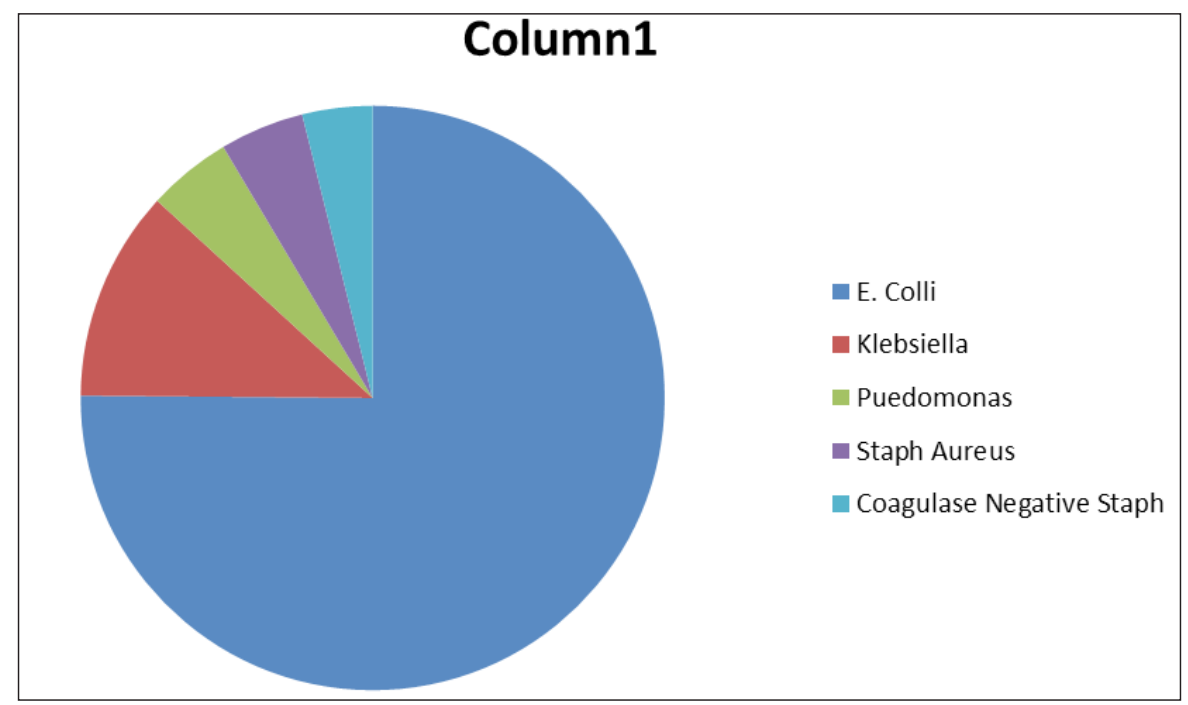

Column 1.Infective Organisms Grown from the Environment in ICU

Table 1.Distribution of Microorganisms in ICU Environment

\begin{tabular}{|c|c|c|c|}
\hline Environment & Total Samples & Infected Samples N (\%) & Organisms N (\% of Infected Samples) \\
\hline Tap knobs (two) & 52 & $13(25 \%)$ & E. coli $-13(100 \%)$ \\
\hline Sinks (two) & 52 & $45(86.5 \%)$ & $\begin{array}{c}\text { E. coli }-28(29 \%) \\
\text { Klebsiella }-13(29 \%) \\
\text { Pseudomonas }-4(9 \%)\end{array}$ \\
\hline Medical tray & 26 & $1(3.8 \%)$ & Pseudomonas - $1(100 \%)$ \\
\hline Ventilator & 26 & $3(11.5 \%)$ & $\begin{array}{l}\text { Pseudomonas -1 (33\%) } \\
\text { Coag. -ve Staph } 2(67 \%)\end{array}$ \\
\hline Bedrail & 26 & $3(11.5 \%)$ & S. aureus $-2(33 \%)$ \\
\hline Disinfectant & 26 & 0 & NA \\
\hline Stethoscope & 26 & $6(23 \%)$ & Coag. -ve Staph - 2 (33\%) \\
\hline Doctor & 26 & 0 & $\mathrm{NA}$ \\
\hline Nurse & 26 & $2(7.7 \%)$ & Klebsiella - 2 (100\%) \\
\hline Water (two sites) & 52 & $52(100 \%)$ & E. coli- $52(100 \%)$ \\
\hline Total & 338 & $125(37 \%)$ & $\begin{array}{c}\text { E. coli - } 93(74.4 \%) \\
\text { Klebsiella - } 15(12 \%) \\
\text { Pseudomonas - } 6(4.8 \%) \\
\text { Coag. -ve Staph }-(4.8 \%) \\
\text { S. aureus - } 5(4 \%)\end{array}$ \\
\hline
\end{tabular}

\section{Discussion}

The past several decades have seen an increased effort in characterizing the epidemiology of healthcare-associated infections and in advancing the knowledge of infectionprevention practices. This is seen more in the high dependency units and intensive care units.

The hospital environment plays a crucial role in exposing patients to various pathogens including bacterial, fungal and viral. These organisms may be found on the hands of caretakers, on the knobs of doors, on keyboards, or in the structure and environment of the room itself, increasing the chances of nosocomial infection. The incidences of the environmental burden of pathogens and subsequently ways to lower the rates of hospital-acquired infections have also been studied. ${ }^{5}$

In the present study, surveillance for presence of infection was carried out in environment samples and samples from healthcare workers twice a week for three months. Different sites in the ICU were included for environmental sampling out of which two were water samples. Two swabs were taken from the hands of two healthcare workers (doctors, nurses, male orderlies) twice a week each week. The water samples from the two taps showed presence of coliforms. Bacterial growth was seen in several samples.

In our study, the commonest organism isolated was $E$. coli (74.4\%) of the positive samples. As all the 52 water samples 
were positive for $E$. coli and it was found in samples from tap knobs and sinks and no other environmental sample, this tends to suggest that the source of $E$. coli was water supply to the ICU. It also highlights the importance of treating water that is supplied to high risk areas like ICUs.

The second commonest organism was Klebsiella which was found mainly in both the sinks and only two positive samples from the healthcare workers (nurses). As there was a prevalence of Klebsiella infection in ICU patients during the period of study, it seems that the source of organism in sinks was from ICU patients whose airway equipment and circuits were washed in these sinks. There were only two samples positive from nurses as there was a very good compliance of hand washing and wearing of gloves by most of the healthcare workers. Compliance rates of healthcare workers using proper hand hygiene before and after patient care activities vary, ranging from $24 \%$ to $89 \%$ (an average of $56.6 \%)^{5}$

The other relatively low occurring organisms in our study were Psuedomonas and coagulase negative S. aureus (4.8\% each of positive samples). Psuedomonas grew on samples from sinks, medical trays and ventilators whereas staphylococcus was found on ventilators, bed rails and diaphragms of stethoscopes. A study by Rogues et al. ${ }^{6}$ examined tap water source as a source of Pseudomonas Aeruginosa in a medical care setting. They screened specimens of patients, tap water and hands of health care workers (HCWs) over a period of six months. A total of 657 tap water samples were collected from 39 faucets and 127 hands of HCWs were sampled. $P$. aeruginosa was found in $11.4 \%$ of 448 tap water samples taken from patients' rooms and $5.3 \%$ of 189 other tap water samples $(p<0.01)$.

Blanc et al. ${ }^{7}$ performed a prospective epidemiological during a nonepidemic period of 1 year. The inner parts of the ICU faucets were swabbed for $P$. aeruginosa. Data were recorded on all patients with at least one culture of a clinical specimen positive for $P$. aeruginosa. The strains were characterized using pulsed gel electrophoresis. They studied five ICUs of a university hospital which were supplied by two separate water distribution network. In $42 \%$ of cases $(56 / 132)$ there were isolates identical to those found in the faucets, with a total of nine different genotypes. Among the nine genotypes isolated from both patients and faucets one of them, the most prevalent, was isolated in the two networks and in 30 cases. The other eight genotypes were recovered almost exclusively from either one (three genotypes in 12 cases) or the other (five genotypes in 12 cases) network and from the patients in the corresponding ICUs.

In our study, there was a markedly high level of infection. It was mainly due to high prevalence of coliform organism in the water of the ICU taps. Also it may be due to the fact that the present study was carried out in patients admitted in the ICU, where both antibiotic consumption and development of resistance is highest among all healthcare settings. It also appears that in ICU setups, ICU environmental infection can spread to the critically ill ICU patients and the infected patients, in turn, can also transmit infection to the ICU environment.

\section{Conclusion}

The ICU environment seems to be a potential reservoir for pathogens. Therefore, strict adherence to environmental infection-control measures is essential to prevent healthcare-associated infections. We recommend:

- Intensive infection-prevention program with emphasis on patient safety, and communication among healthcare workers should be strictly implemented in an ICU to decrease the rate of infection.

- Caution should be taken in any ICU that plumbing water, and air filtration systems are closely monitored. The water given to high-risk areas should probably be treated further (e.g., by ultra violet or reverse osmosis) before use.

- The equipment used on the patient should be immediately treated with disinfectants and only after adequate exposure to the disinfectant be taken to the ICU sink for washing.

- Appropriate hand hygiene is vital to patient care and is extremely cost-effective. The hand hygiene task force should be created to educate and monitor the activities of healthcare workers.

- Incorporating rapid diagnostic strategies in the microbiology laboratories and antimicrobial susceptibility testing will reduce indiscriminate antibiotic usage and help combat ICU infections.

- A multidirectional approach, including continuing staff education, minimizing risk factors, and implementing guidelines established by national committees.

\section{Conflict of Interest: None}

\section{References}

1. Joint Commission Resources. Improving care in the ICU. 1st edition. Oakbrook Terrace (IL): Joint Commission Resources 2004.

2. Society of Critical Care Medicine. Critical care units: $A$ descriptive analysis. $1^{\text {st }}$ edition. Des Plaines (IL): Society of Critical Care Medicine 2005.

3. Warren DK, Shukla SJ, Olsen MA et al. Outcome and attributable cost of ventilator-associated pneumonia among intensive care unit patients in a suburban medical center. Crist Care Med 2003; 31(5): 1312-17.

4. Gandhi TN, De Pestel DD, Collins CD et al. Managing antimicrobial resistance in intensive care units. Crit Care Med 2010; 38(8Suppl): S315-23. 
5. Barsanti MC, Woeltje KF. Infection prevention in intensive care units. Infect Dis Clin N Am 2009; 2370325.

6. Rogues AM, Boulestreau H, Lashera A et al. Contribution of tap water to patient colonization with Pseudomonas aeruginosa in a medical intensive care unit. J Hosp Infect 2007; 67: 72-78.
7. Blanc DS, Nahimana I, Petignat $C$ et al. Faucets as a reservoir of endemic Pseudomonas aeruginosa colonization/infections in intensive care units. Intensive Care Med 2004 Oct; 30(10): 1964-68. Epub 2004 Jul 15.

Date of Submission: 2018-04-11 Date of Acceptance: 2018-04-18 